\title{
KINEMATIC SYNTHESIS USING TREE TOPOLOGIES
}

\author{
Edgar Simo-Serra ${ }^{\mathrm{a}}$, Alba Perez-Gracia ${ }^{\mathrm{b}, *}$ \\ ${ }^{a}$ Institut de Robotica i Informatica Industrial (CSIC-UPC), Llorens i Artigas 4-6, 08028 \\ Barcelona, Spain \\ ${ }^{b}$ Department of Mechanical Engineering, Idaho State University, Pocatello, Idaho 83209, \\ $U S A$
}

\begin{abstract}
This paper presents a methodology for the description and finite-position dimensional synthesis of articulated systems with multiple end-effectors. The articulated system is represented as a rooted tree graph. Graph and dimensional synthesis theories are applied to determine when exact finite-position synthesis can be performed on the tree structures by considering the motion for all the possible subgraphs. Several examples of tree topologies are presented and synthesized. This theory has an immediate application on the design of novel multi-fingered hands.
\end{abstract}

Keywords: kinematic synthesis, graph theory, tree articulated systems

\section{Introduction}

Kinematic synthesis theory, in which an articulated system is designed to meet certain motion specifications, has been applied to open and closed linkages. In the dimensional synthesis problem, a given topology for an articulated system is dimensioned in order for its workspace to fit a given task. Finite-position dimensional synthesis of planar linkages was developed early, see [1, 2, 3, 4]. Dimensional synthesis of spatial articulated systems has targeted mostly serial chains. The first methods used [5, 6], based on

*Corresponding author. Phone number 0012082825655

Email addresses: esimo@iri.upc.edu (Edgar Simo-Serra), perealba@isu.edu ( Alba Perez-Gracia) 
geometric constraints and vector loop equations, were successful for synthesizing simple systems. More recently, methods based on robot kinematic equations [7, 8] allowed to formulate design equations for more complex systems, still limiting the application to serial chains, and with limitations in the solution process [9]. Some examples of dimensional synthesis applied to parallel robots can be found in [10, 11, 12].

Type or structural synthesis [13, 14] includes a systematic classification of the linkage type in the synthesis process, which is based on graph theory in many cases. Graph theory has been used for a long time in the analysis and type synthesis of linkages, see early research by Woo [15], Huang and Soni [16], Manolescu [17], Freudenstein and Maki [18], and more recently Tsai [19], Mruthyunjaya [20], and Lu, Mao et al. [21], 22]. Additionally, Chuang and Lee [23] have used structural synthesis for the design of finger mechanisms.

The analysis of articulated systems with a tree structure has also generated some research. Selig [24] mentions tree-structured mechanisms and models them as rooted trees following [25]. His work includes basic definitions and the application to kinematic and dynamic analysis. Chen et al. [26] perform the analysis of tree-type geometries for applications in modular robots. Song and Amato [27] apply the analysis of tree-like articulated systems to folding. Jain [28] uses tree graphs for the dynamic analysis of multi-body systems; tree-systems also appear describing dynamic systems in Garcia de Jalon [29]. Tree articulated systems and their graph representation are also studied in [30] for the analysis and control of mechanical systems, where they are named forking linkages. Tischler et al. [31, 32] apply graph theory for the structural synthesis of kinematic chains with applications to robot hands. However, no applications or methodology exist, to the knowledge of the authors, for the dimensional synthesis of articulated systems with a tree structure.

Even though little literature is devoted to these systems when compared to their serial or parallel counterparts, tree articulated systems are widely used in robotic multi-fingered hands. Locomotive structures for mobile robots could also be modeled as tree-like systems. For this research, the kinematic design of multi-fingered robotic grippers is the primary application.

The advantage of having a methodology for the dimensional synthesis of a set of fingers lies in the possibility of defining simultaneous manipulation and grasping actions for the whole hand. Aside from underactuated robotic 
hands (see [33] for a review and [34] for recent results), which present little dexterity, the design of robotic hands has been performed from an anthropomorphic point of view. See recent reviews of different applications in [35, 36]. In addition to this, modular fingers have been designed that able to perform certain grasping actions and are later integrated in a hand [37. The development of a theory for the use of dimensional kinematic synthesis on tree topologies aims to create a tool for new designs that can be applied, for instance, in many areas of human-robot interaction.

This paper focuses on developing a general methodology for the finiteposition dimensional synthesis problem, in particular exact dimensional synthesis, applied to articulated systems with a tree structure. A tree-structured articulated system has a base (a grounded link), some common joints, and multiple end-effectors, each one of them corresponding to a separate branch.

The input data of the synthesis algorithm is a finite set of rigid-body positions for each of the multiple end-effectors and a selected tree topology, which is modeled using rooted tree graphs as explained in Section 2, After the substitution of kinematic chains, a compact rooted graph is obtained. This graph must be checked for solvability. In case of obtaining a non-solvable graph, an equivalent graph must be found. These two steps are developed in Sections 4 and 5 .

For the resulting solvable rooted tree graph, the forward kinematics equations of relative displacements for each serial chain are computed using dual quaternions. The theory is introduced in Section 3. The synthesis of spatial serial chains for up to five degrees of freedom using this technique was developed in [38]. In this case, each serial chain corresponds to one branch, including the common joints. The dependency among the different serial chains allows the extension of the exact synthesis to articulated systems with a high number of degrees of freedom, and to tasks defined by a high number of positions. The extension of the kinematic synthesis to tree topologies is presented in Sections 6 and 7, and the matrix representation can be found in Section 8 .

Each serial chain yields a set of equations and all the sets are solved simultaneously. Due to the high dimension and degree of the obtained system of equations, a numerical solver is required. As a last step, the solution is used to dimension the substituted kinematic chains.

Three examples are included in Section 9. The first one is a possible application to a multi-fingered robotic hand, for which numerical solutions have been found [39]. The second example is a simple PR-(R,P) tree struc- 
ture, which can be solved analytically and is used to illustrate the process. The last example includes a numerical task for an RR-(RR,R,R) topology, and one of the multiple solutions obtained is presented. For ease of comprehension, an overview of the nomenclature used in this paper is presented in Table 1.

The development of a dimensional synthesis method for tree articulated systems is a first step towards a new design tool for multi-fingered robotic hands. The non-anthropomorphic hands obtained, which can perform human tasks if so designed, could be used for specific robotics applications, and in particular for human-robot interaction or cooperative tasks.

Table 1: Nomenclature used in this paper

\begin{tabular}{cl}
\hline Symbol & Description \\
\hline $\mathbf{S}$ & A vector. \\
{$[M]$} & A matrix. \\
$\hat{a}$ & A dual number. \\
$\hat{Q}$ & A dual quaternion. \\
$\mathbf{S}$ & Plucker coordinates of a line; also a screw. \\
$\$^{*}$ & Smallest subalgebra of $\mathfrak{s e}(3)$ containing all the possible infinites- \\
& imal mechanical liaison between two rigid bodies. \\
$\$$ & An ordered screw surface. \\
$\mathfrak{L}$ & Linkage locus space. \\
\hline
\end{tabular}

\section{Rooted Tree Graph Representation}

Articulated systems with a tree topology can be modeled using graph theory. This allows for a compact representation of the structure, the identification of key paths in the system, and the realization of some operations that help simplify the synthesis process.

The use of graphs in order to represent mechanisms was proposed by Crossley [40]. Tsai's methodology [19] is followed in this paper. It consists of identifying the joints with the edges and the links with the vertices of the graph. The different types of joints (revolute, prismatic, spherical, etc.) are 
indicated using their common abbreviation ( $\mathrm{R}, \mathrm{P}, \mathrm{S}$, etc.) on the edges of the graph. See for instance Figure 1 .

For their use in the kinematic synthesis process, the mechanisms are to be represented always as rooted graphs, the root vertex being fixed with respect to the reference system.

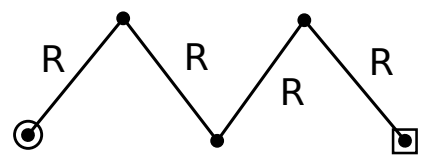

Figure 1: Graph representation of a 4R serial manipulator. The circle indicates the root vertex and the square indicates the end-effector.

A rooted connected graph representing a kinematic structure shall be denoted as $G(V, E)$, with a set of $v$ vertices $\mathrm{V}$ and a set of $e$ edges $\mathrm{E}$ connecting the vertices. The vertices represent the rigid bodies and the edges represent the joints connecting adjacent bodies. Besides the root vertex, a number of vertices will be given the special characteristic of being considered end-effectors.

A graph representing a 4R manipulator can be seen in Fig. 1. The four edges correspond to the four revolute joints. The root vertex is marked with a circle around the vertex and the end-effector is indicated with a square mark.

In tree topologies, a vertex can be connected to several edges defining several branches. The degree of a vertex is the number of edges that are connected to it, which in this case would be equivalent to the number of joints connected to it. Notice that a tree articulated system will always have links that are ternary or above; those can be identified in the graph as a vertex spanning several edges.

\subsection{Contraction}

After constructing the initial graph representation of a linkage, the next step is to contract the graph. The graph is contracted so that each edge represents the set of joints of a kinematic serial chain instead of an individual kinematic joint.

The $4 \mathrm{R}$ manipulator seen in Fig. 1 would be contracted to a single edge representing the serial revolute joints connecting the root vertex and the end- 
effector. Contraction removes all vertices with a degree of 2 ; notice that all vertices with a degree of 1 that are not the root vertex are end-effectors.

\subsection{Setting the Root Node}

The special characteristic of the root node is that it indicates that the rigid body associated with it is immobile in the reference system. Essentially it marks the fixed reference system. However, the root node can be set at any other end-effector by performing a transformation. Consider the positions $\mathbf{P}_{i}$ as rigid transformations associated to the end-effector $i$ where $i=1$ is the root node, then the inversion of the root from $i=1$ to $i=j$ is

$$
\mathbf{P}_{i}^{*}=\mathbf{P}_{j}^{-1} \mathbf{P}_{i}
$$

where $\mathbf{P}_{i}^{*}$ would be the new end-effector positions relative to the new root $j$.

Changing the root node can be useful for solving particular systems, as will be shown in the examples.

\subsection{Rooted Graphs for Tree Topologies}

Let $T(V, E)$ be a contracted rooted tree graph, with a set of $v$ vertices $\mathrm{V}$ and a set of $e$ edges $\mathrm{E}$, obtained from a rooted connected graph $G\left(V_{r}, E_{r}\right)$. It is assumed that there are no loops in the contracted rooted tree graph. For synthesis purposes, loops can be substituted with equivalent serial chains in a process called reduction, which is explained in Section 5. Then the following relations exist:

1. $e=v-1$

2. Between any two vertices $i$ and $j$ where $i \neq j$ there exists only one path.

3. The only vertices with a degree of 1 will be either the graph root or an end-effector.

4. There are no vertices of degree 2 .

\section{Forward Kinematics for Tree Topologies}

The forward kinematics for a serial kinematic chain can be written using the representation-agnostic exponential map [41], 


$$
\hat{Q}(\hat{\boldsymbol{\theta}})=\left(\prod_{i=1}^{n} e^{\frac{\hat{\theta}_{i}}{2} \mathbf{S}_{i}}\right) \hat{g}
$$

where $\hat{\theta}_{i}=\theta_{i}+\epsilon d_{i}$ are the joint parameters written as a dual number, with $\theta_{i}$ and $d_{i}$ being the rotation about and displacement along the screw axis respectively; $\hat{g}$ is the transformation from the fixed frame to the endeffector at a reference configuration; and $S_{i}$ is the $i^{\text {th }}$ joint axis at a reference configuration.

The term $\hat{g}$ can be eliminated by considering the forward kinematics of relative displacements with respect to this reference configuration, measured from the fixed frame,

$$
\hat{Q}(\boldsymbol{\Delta} \hat{\boldsymbol{\theta}})=\prod_{i=1}^{n} e^{\frac{\Delta \hat{\theta}_{i}}{2} S_{i}}
$$

where $\Delta \hat{\theta}=\theta-\theta_{0}+\epsilon\left(d-d_{0}\right)$, with $\theta_{0}$ and $d_{0}$ being the values of the joint parameters at the reference configuration.

For a linkage with a tree topology, a set of forward kinematics equations can be written for each of the branches, considering the common joints in all of them,

$$
\hat{Q}_{i}(\boldsymbol{\Delta} \hat{\boldsymbol{\theta}})=\underbrace{\prod_{j=1}^{k_{i}} e^{\frac{\Delta \hat{\theta}_{j}}{2}} \mathrm{~S}_{j}}_{\text {common }} \underbrace{\prod_{j=k_{i}+1}^{n_{i}} e^{\frac{\Delta \hat{\theta}_{i, j}}{2} \mathrm{~S}_{i, j}}}_{\text {branch }}, \quad i=1, \ldots, b
$$

where the number of common joints for a given branch $i$ is indicated by $k_{i}$, and the number of end-effectors, or branches, is indicated by $b$. Each branch or serial chain has a total of $n_{i}$ joints, including the common joints shared with other branches.

\section{Linkage Locus Space}

For the most general case of a tree topology, where the joint axes can be arbitrarily positioned in space, it is important to define the minimum 
subgroup of the special Euclidean group $S E(3)$ able to contain the full motion of each end-effector. In the rest of this article, $S E(3)$ will denote the special Euclidean group and $\mathfrak{s e}(3)$ will denote its corresponding Lie algebra.

For a given serial chain with $n$ joint axes $\mathrm{S}_{i}$, the workspace of relative motion can be defined as

$$
W=\left\{\mathbf{w} \mid \prod_{i=1}^{n} e^{\frac{\Delta \hat{\theta}_{i}}{2} S_{i}}-\mathbf{w}=0, \quad \forall \hat{\boldsymbol{\theta}}\right\} .
$$

However, for performing synthesis, an extended version of the workspace needs to be considered; the potential workspace of a generic mechanism topology, as opposed to the particular workspace of a specific mechanism. In order to define where the generic mechanism topology lies, the Linkage Locus Space is defined.

Let $\$_{J}$ be the screw system corresponding to a given joint or set of joints $J$, and let $\$_{J}^{*}$ be the smallest subalgebra of $\mathfrak{s e}(3)$ containing all the possible infinitesimal mechanical liaisons 42 between two rigid bodies of a serial chain connected by that joint or set of joints. In general, this is calculated for consecutive rigid bodies separated by a joint. However, in the case of having constraints defined by relationships between joint parameters for two or more consecutive rigid bodies in a serial chain, this cannot be calculated as the mechanical liaison of individual joints.

For example $\$_{P}^{*}$ would be the smallest subalgebra that contains all possible infinitesimal mechanical liaisons of the form,

$$
\mathrm{S}_{P}=\lambda(0 ; \mathbf{s}),
$$

which in this case would be $\$_{P}^{*}=\mathbb{R}^{3}$. Consider the helicoidal joint, which has the form

$$
\mathrm{S}_{H}=\lambda(\mathbf{s} ; \mathbf{r} \times \mathbf{s}+h \mathbf{s}) .
$$

The smallest subalgebra containing its generic version is $\$_{H}^{*}=\mathfrak{s e}(3)$.

Special relationships can also be considered using explicit calculation. As an example, consider the case of a revolute joint followed by a cylindric joint forming an RC chain, where both joints have the same rotation axis with opposite angles. The coordinates of each joint would be 


$$
\begin{aligned}
& \mathrm{S}_{R}=\theta\left(\mathbf{s} ; \mathbf{r}_{R} \times \mathbf{s}\right) \\
& \mathrm{S}_{C}=-\theta\left(\mathbf{s} ; \mathbf{r}_{C} \times \mathbf{s}\right)+d(0 ; \mathbf{s})
\end{aligned}
$$

with $\theta$ and $d$ being the joint parameters. When considering all the displacements generated by the RC subchain,

$$
W_{R C}=\left\{\mathbf{w} \mid e^{\frac{\Delta \hat{\theta}_{R}}{2} S_{R}} e^{\frac{\Delta \hat{\theta}_{C}}{2} S_{C}}-\mathbf{w}=0, \quad \forall \hat{\boldsymbol{\theta}}\right\},
$$

which expand to the form

$$
\mathrm{S}_{R C}=\left(0 ; \theta\left(\mathbf{r}_{R} \times \mathbf{s}-\mathbf{r}_{C} \times \mathbf{s}\right)+d \mathbf{s}\right),
$$

the smallest subalgebra of $\mathfrak{s e}(3)$ containing all possible screws of the form $\mathrm{S}_{R C}$ in this case would be $\mathbb{R}^{3}$.

Considering the entire chain, it is possible to define the Linkage Locus Space $\mathfrak{L}$ as the smallest subalgebra of $\mathfrak{s e}(3)$ corresponding to the smallest subgroup containing the generic workspace $S$ for the given topology,

$$
\mathbf{S}=\left\{\mathbf{w} \mid \prod_{i=1}^{n} e^{\frac{\Delta \hat{\theta}_{i}}{2} \mathbf{S}_{i}}-\mathbf{w}=0, \forall \hat{\boldsymbol{\theta}}, \forall \mathrm{S}_{i} \in \$_{i}^{*}\right\}
$$

This can be calculated directly by finding the smallest subalgebra that contains $\mathrm{S}$ or, given all the subalgebras of all the joints of the serial chain, by using the closure of subalgebras

$$
\mathfrak{L}=\$_{1}^{*} \odot \$_{2}^{*} \odot \cdots \odot \$_{n}^{*}
$$

Proof. Firstly w can be expanded by using the well-known Campbell-BakerHausdorf series, 


$$
\begin{aligned}
& \log (\mathbf{w})=\log \left(\prod_{i=1}^{n} e^{\frac{\Delta \hat{\theta}_{i}}{2} \mathrm{~S}_{i}}\right) \\
&=\frac{\Delta \hat{\theta}_{1}}{2} \mathrm{~S}_{1}+\frac{\Delta \hat{\theta}_{2}}{2} \mathrm{~S}_{2}+\cdots+\frac{\Delta \hat{\theta}_{n}}{2} \mathrm{~S}_{n}+ \\
& \frac{1}{8}\left(\Delta \hat{\theta}_{1} \Delta \hat{\theta}_{2}\left[\mathrm{~S}_{1}, \mathrm{~S}_{2}\right]+\Delta \hat{\theta}_{1} \Delta \hat{\theta}_{3}\left[\mathrm{~S}_{1}, \mathrm{~S}_{3}\right]+\cdots\right)+ \\
& \frac{1}{96}\left(\Delta \hat{\theta}_{1}^{2} \Delta \hat{\theta}_{2}\left[\mathrm{~S}_{1},\left[\mathrm{~S}_{1}, \mathrm{~S}_{2}\right]\right]+\right. \\
&\left.\Delta \hat{\theta}_{1} \Delta \hat{\theta}_{2}^{2}\left[\mathrm{~S}_{1},\left[\mathrm{~S}_{1}, \mathrm{~S}_{3}\right]\right]+\cdots\right)+\cdots
\end{aligned}
$$

The closure of subalgebras from Eqn. (12) is a vector subspace containing all the finite Lie products of the form,

$$
\mathfrak{L}=<\mathrm{S}_{1} \mathrm{~S}_{2} \cdots \mathrm{S}_{n}\left[\mathrm{~S}_{1}, \mathrm{~S}_{2}\right] \cdots\left[\mathrm{S}_{1},\left[\mathrm{~S}_{1}, \mathrm{~S}_{2}\right]\right] \cdots>
$$

It is straightforward to see that a linear combination of elements from Eqn. (14) can be found for each element of $\log (\mathbf{w})$ in the form of,

$$
\left(\frac{\Delta \hat{\theta}_{1}}{2} \frac{\Delta \hat{\theta}_{2}}{2} \cdots \frac{\Delta \hat{\theta}_{n}}{2} \frac{\Delta \hat{\theta}_{1} \Delta \hat{\theta}_{2}}{8} \frac{\Delta \hat{\theta}_{1} \Delta \hat{\theta}_{3}}{8} \cdots\right)
$$

From this it can be deduced that,

$$
\log (\mathbf{w}) \in \mathfrak{L}, \quad \forall \mathbf{w} \in S
$$

and thus,

$$
\log (\mathrm{S}) \leq \mathfrak{L}
$$

proving that $\log (\mathrm{S})$ is closed under the Lie bracket and is therefore a subalgebra of $\mathfrak{s e}(3)$. For simplicity let us take two arbitrary elements of $\log (\mathrm{S})$ in the form of,

$$
X=X_{1}+X_{2}+\cdots+X_{n}+\frac{1}{2}\left[X_{1}, X_{2}\right]+\cdots
$$


The Lie bracket of two elements, which denoted as $X$ and $X^{*}$ respectively, can be expanded as,

$$
\begin{array}{r}
{\left[X_{1}+X_{2}+\cdots+X_{n}+\frac{1}{2}\left[X_{1}, X_{2}\right]+\cdots,\right.} \\
\left.X_{1}^{*}+X_{2}^{*}+\cdots+X_{n}^{*}+\frac{1}{2}\left[X_{1}^{*}, X_{2}^{*}\right]+\cdots\right]= \\
{\left[X_{1}, X_{1}^{*}\right]+\left[X_{1}, X_{2}^{*}\right]+\cdots+\left[X_{2}, X_{1}^{*}\right]+\cdots+} \\
\frac{1}{2}\left(\left[\left[X_{1}, X_{2}\right], X_{1}^{*}\right]+\left[\left[X_{1}, X_{2}\right], X_{2}^{*}\right]+\cdots\right)+\cdots
\end{array}
$$

where each element in the summation belongs to $\mathfrak{L}$.

The subalgebras $\$_{i}^{*}$ are also contained in $\log (\mathrm{S})$. As $\mathfrak{L}$ is the smallest subalgebra containing all $\$_{i}^{*}, \log (S) \leq \mathfrak{L}$, and $\log (\mathrm{S})$ contains all $\$_{i}^{*}$, it can be established that,

$$
\log \left(\prod_{i=1}^{n} e^{\$_{i}^{*}}\right)=\log (\mathrm{S})=\mathfrak{L}=\$_{1}^{*} \odot \$_{2}^{*} \odot \cdots \odot \$_{n}^{*}
$$

Therefore, in order to calculate $\operatorname{dim}(\mathrm{S})$, it is possible to calculate instead $\operatorname{dim}(\mathfrak{L})$ by means of iteratively adding successive higher order Lie brackets for each subalgebra $\$_{i}^{*}$, until all the Lie brackets are linear products of the previously found Lie brackets.

As shown in the next section, the expressions developed here can be used to define the dimension of the space in which all possible tasks for mechanisms of a given topology can be found. This is important for both being able to define a task that is feasible for a given topology and also being able to determine when exact synthesis can be performed.

\section{Reduced Tree Topology for Exact Synthesis}

The main objective of reduction is to find an equivalent tree graph for performing exact dimensional kinematic synthesis. At the end of the process, the remaining serial chains or edges must have a finite number of solutions for dimensional synthesis, while keeping the motion constraints of the original mechanism. An example of a complex graph representing a kinematic 


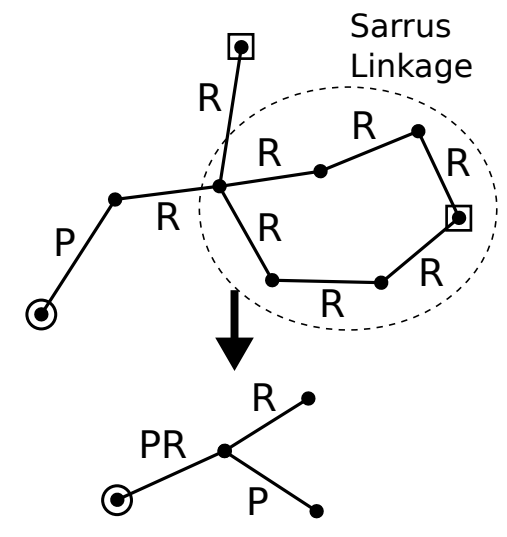

Figure 2: Reduction of a complex kinematic structure with a Sarrus linkage to a tree graph.

structure being contracted and reduced to a rooted tree graph can be seen in Fig. 2.

In the case of open-loop chains, the mobility of the loops can be calculated using the well-known Chebyshev-Kutzbach-Grübler (CKG) equation:

$$
F=\lambda(n-j-1)+\sum_{i=1}^{j} f_{i}
$$

where $F$ is the number of degrees of freedom of the mechanism, $\lambda=\operatorname{dim}(W)$ [10] is the number of allowed degrees-of-freedom of the space in which the end-effector moves, $n$ is the number of links, $j$ is the number of joints, and $f_{i}$ indicates the degrees of freedom of joint $i$.

However, this mobility does not take into account the actual structure of the workspace. With a mobility of 4 the workspace could be redundantly defined, as would be the case of four prismatic joints. Therefore the dimension of the linkage locus space must also be taken into account.

The number of redundant degrees-of-freedom $r$ of a serial mechanism may be calculated by

$$
r=\sum_{k=1}^{j} f_{k}-\operatorname{dim}(\mathfrak{L}),
$$


where $f_{k}$ denotes the number of degrees of freedom of joint $k$.

If $r>0$ the mechanism is considered to have redundant degrees-offreedom. For a given relative transformation there will be an entire subspace of joint parameters that will perform the transformation. These mechanisms are not suitable for exact dimensional synthesis, as there will be a subspace of solutions and not a finite number of solutions.

Chains without redundant degrees of freedom, able to move anywhere in the Linkage Locus space $\mathfrak{L}$, may have subspaces of solutions too. The degrees of freedom can be represented as the ordered screw surface $\$$ of a mechanism topology as:

$$
\begin{aligned}
\$ & =\left[\begin{array}{l}
\$_{1} \$_{2} \cdots \$_{n}
\end{array}\right] \\
& =\$_{1} \oplus \$_{2} \oplus \cdots \oplus \$_{n}
\end{aligned}
$$

where $\$_{i}$ is the Lie subalgebra of joint $i$.

In order for the kinematic synthesis to have a finite number of solutions the kinematic chain must comply with

$$
\operatorname{dim}(\$)<\operatorname{dim}(\mathfrak{L})
$$

The combined condition that the serial chains must comply with to be able to perform exact kinematic synthesis becomes,

$$
\sum_{k=1}^{j} f_{k}=\operatorname{dim}(\$)<\operatorname{dim}(\mathfrak{L}) .
$$

Additionally, $S E(3)$ is formed by $S O(3) \rtimes \mathbb{R}^{3}, \mathbb{R}^{3}$ being a normal subgroup. The Lie bracket of any vector from the subspace of $\mathfrak{s e}(3)$ with any vector of $\mathbb{R}^{3}$ belongs to $\mathbb{R}^{3}$. In order to perform exact kinematic synthesis, $\mathbb{R}^{3}$ must not be a subspace of the ordered screw surface,

$$
\nexists \mathbf{s} \mid\left(\mathbf{s} ; \mathbf{s}^{0}\right) \in \$, \forall \mathbf{s}^{0}
$$

Kinematic serial chains that either fully determine the Linkage Locus space $\mathfrak{L}$ or have redundant degrees of freedom must be substituted by other 
chains that preserve $\mathfrak{L}$ while having a finite number of kinematic synthesis solutions. This can be seen as solving a more constrained problem in order to obtain intermediate positions, which are then used to solve the original problem, albeit it will not have a finite number of solutions.

The process of substituting serial chains that are not suitable for exact dimensional synthesis is not straightforward when there are many constraints applied to the kinematic chain. For non-complex cases such as the $4 \mathrm{P}$ serial chain it is simple to see that it must be substituted for the $2 \mathrm{P}$ serial chain, which shares the $\mathfrak{L}=\mathbb{R}^{3}$ Linkage Locus space with no redundant degrees of freedom.

\subsection{Parallel Mechanism Equivalence}

To be able to convert the graph to a tree graph the loops must be replaced with their non-loop equivalents. Refer to the study of the mobility of singleloop kinematic chains done by Rico and Ravani [42] for a more in depth study of types of kinematic loops.

Single kinematic loops can be split into two paths, corresponding to the clockwise and counterclockwise directions between two rigid bodies. In order to study the mobility, the intersection of the subalgebras corresponding to both paths must be analyzed. Depending on the class of the loops, different steps must be taken to substitute the loop for a non-loop equivalent.

Trivial kinematic loops are the simplest class to handle. In this case the intersection is either the clockwise or counterclockwise path of the loop. A path yielding the intersection is called the dominant path; the other path is to be removed, as the restrictions imposed by the dominant path are in fact what are determining the workspace of the mechanism.

Exceptional kinematic loops have an intersection of subalgebras that is not trivial and is not equal to neither path. The exceptional kinematic chain loop can be substituted for the subalgebra corresponding to the intersection which may not always have a simple equivalence. Fanghella and Galletti [43] find many equivalences of intersections using group theory, and Rico and Ravani [42] do the same using Lie algebras. However, finding the equivalent open kinematic chain is not always a simple task, depending on the mobility class of the loop.

Kinematic chains with partitioned mobility can be removed from the graph, that is, the edge representing the chain can be removed and both vertices merged into a single one, as they do not have relative motion. 
The remaining cases, which correspond to paradoxical kinematic chains of classes 1 and 2, must be handled on a case-to-case basis, as there is no general solution.

This process is to be repeated until there are no loops in the graph and it becomes a tree graph. The equivalent serial chains also need to comply with the serial chain requirements from Eqn. (25).

The reduction of a Sarrus linkage to an equivalent prismatic joint 44] can be seen in Fig. 2. The Sarrus linkage is formed by two 3R branches with a set of constraints that give it the same workspace as a single prismatic joint. The problem can then be solved considering the Sarrus linkage to be a prismatic joint. Afterwards the motion of the prismatic joint can be used to generate a Sarrus linkage.

\section{Kinematic Synthesis}

In this paper, the focus is on the exact dimensional kinematic synthesis for a finite set of positions. Dimensional kinematic synthesis seeks to find the location and orientation of all joint axes in order for each of the endeffectors to perform a given set of displacements. In this section, an overview of the process for serial chains is presented, and in the next chapter it shall be extended to tree topologies.

\subsection{Design Equations}

Using the relative forward kinematics from Eqn. (3), the design equations for a serial kinematic chain to reach $m$ finite positions are

$$
\hat{P}_{1 j}=\prod_{i=1}^{n} e^{\frac{\Delta \hat{\theta}_{i}^{j}}{2} \mathrm{~S}_{i}}, \quad j=2, \ldots, m,
$$

where $j=1$ is considered the reference configuration, and $\hat{P}_{1 j}=\hat{P}_{j} \hat{P}_{1}^{-1}$.

This creates a set of design equations that can be solved in order to reach the set of relative displacements $\hat{P}_{1 j}$. Therefore the output of solving the design equations for a given task are the joint axes in the reference position and the relative displacements of the joints to each of the remaining $m-1$ positions.

In addition to the design equations, different equations can be included, for example relationships between joint axes would be considered as additional constraints $c$. Furthermore, the design equations can be modified to handle velocities and accelerations as done in [45]. 


\subsection{The Synthesis Task}

To be able to create the synthesis design equations from Eqn. (27), the number of variables and equations in the system must be accounted for. Two types of variables are considered: structural and joint parameters. Joint parameters are those variables parameterizing the motion about the joints and depend on the number of end-effector poses. Structural parameters define the location of the joints and depend only on the number and types of the joints. The total number of variables $n_{x}$ will be

$$
n_{x}=(m-1) n_{j}+n_{s}
$$

where $m$ is the number of task positions, $n_{j}=\operatorname{dim}(\$)$ is the number of joint variables, and $n_{s}$ is the number of structural parameters.

The complete set of equations includes design equations and constraints. The design equations come from Eqn. (27) and depend on the number of end-effector positions, while here it is assumed that constraints do not. The total number of independent equations $n_{f}$ will be,

$$
n_{f}=(m-1) d+c
$$

where $d=\operatorname{dim}(\mathfrak{L})$ and $c$ is the number of constraints.

For the system to have a finite number of solutions, $n_{x}=n_{f}$ must be imposed for the task. From there the number of absolute positions needed can be obtained,

$$
m=\frac{n_{s}-c}{d-n_{j}}+1
$$

where all the equations are assumed to be independent.

This leaves he number of structural parameters $n_{s}$ to be calculated. While this may seem like a simple task, it is not always straightforward. Generally any unconstrained screw joint as the one in Eqn. (7) will have 7 parameters: $h \in \mathbb{R}, \mathbf{s} \in \mathbb{R}^{3}$, and $\mathbf{s}^{0} \in \mathbb{R}^{3}$ with two constraints $\mathbf{s} \cdot \mathbf{s}=1$ and $\mathbf{s} \cdot \mathbf{s}^{0}=0$, totaling 5 independent parameters. An unconstrained general revolute joint has 4 parameters as $h=0$. An unconstrained general prismatic joint has 2 parameters as they can be represented by lines at infinity with the form $(0 ; \mathbf{s})$ with $\mathbf{s} \cdot \mathbf{s}=1$. 
Table 2: Design parameters for different serial chains

\begin{tabular}{cccccccc}
\hline Linkage & $n_{j}$ & $n_{s}$ & $d$ & $m$ & $m^{R}$ & $m^{T}$ & $\mathfrak{L}$ \\
\hline $\mathrm{P}$ & 1 & 2 & 3 & 2 & 1 & 2 & $\mathbb{R}^{3}$ \\
$\mathrm{R}$ & 1 & 4 & 6 & $1 \frac{4}{5}$ & 2 & 3 & $S E(3)$ \\
$\mathrm{H}$ & 1 & 5 & 6 & 2 & 2 & $3 \frac{1}{2}$ & $S E(3)$ \\
$\mathrm{C}$ & 2 & 4 & 6 & 2 & 2 & 5 & $S E(3)$ \\
$\mathrm{T}$ & 2 & 5 & 6 & $2 \frac{1}{4}$ & 5 & 6 & $S E(3)$ \\
$\mathrm{E}$ & 3 & 2 & 6 & $1 \frac{2}{3}$ & 2 & $\infty$ & $S E(3)$ \\
$\mathrm{S}$ & 3 & 3 & 6 & 2 & $\infty$ & $\infty$ & $S E(3)$ \\
$\mathrm{PP}$ & 2 & 2 & 3 & 3 & 1 & 3 & $\mathbb{R}^{3}$ \\
$\mathrm{RP}$ & 2 & 6 & 6 & $2 \frac{1}{2}$ & 2 & 7 & $S E(3)$ \\
$\mathrm{RR}$ & 2 & 8 & 6 & 3 & 5 & 9 & $S E(3)$ \\
$\mathrm{PPR}$ & 3 & 6 & 6 & 3 & 2 & $\infty$ & $S E(3)$ \\
$\mathrm{PRP}$ & 3 & 8 & 6 & $3 \frac{2}{3}$ & 2 & $\infty$ & $S E(3)$ \\
$\mathrm{PRR}$ & 3 & 10 & 6 & $4 \frac{1}{3}$ & 5 & $\infty$ & $S E(3)$ \\
$\mathrm{RRR}$ & 3 & 12 & 6 & 5 & $\infty$ & $\infty$ & $S E(3)$ \\
\hline
\end{tabular}


Table 2 shows various sets of kinematic chains. The constraints are considered implicitly as part of the structural parameters $n_{s}$. Different combinations of joints and constraints can modify the number of parameters. A striking example would be that two general prismatic joints PP have a total of 2 parameters and not 4 as expected. This happens whenever two prismatic joints are consecutive in a kinematic serial chain, as their directions of motion span a plane.

There is no general rule for calculating the number of structural parameters $n_{s}$. However, the design equations Eqn. (27) can always be expanded and checked for variable dependencies.

\subsection{Task Degeneration}

The $m$ design equations obtained using Eqn. (30) are not necessarily independent. In fact it is possible for the system of equations to degenerate into two subsystems where one is overdetermined and the other has an infinite number of solutions.

The special Euclidean group $S E(3)$ can be seen as the semi-direct product of two subgroups, $S E(3)=S O(3) \rtimes \mathbb{R}^{3}$. The same technique used to obtain Eqn. (30) can be restricted to the subgroup $S O(3)$ in order to determine the number of rotations needed for the change of orientation of the system. The number of variables $n_{x}^{R}$ becomes,

$$
n_{x}^{R}=\left(m^{R}-1\right) n_{j}^{R}+n_{s}^{R}
$$

where $m^{R}$ is the number of orientations, $n_{j}^{R}$ the number of joints with a revolute component, and $n_{s}^{R}$ the number of structural parameters for the rotations.

The number of independent equations corresponding to rotations, $n_{f}^{R}$, can be defined by

$$
n_{f}^{R}=\left(m^{R}-1\right) d^{R}+c^{R},
$$

where $d^{R}=\operatorname{dim}(\mathfrak{L} \cap \mathfrak{s o}(3))$ and $c^{R}$ is the number of constraints affecting the rotational parameters in the design equations.

Hence, it is possible to define the number of independent orientations $m^{R}$ needed to fully define the rotational component of design equations by making $n_{x}^{R}=n_{f}^{R}$, 


$$
m^{R}=\frac{n_{s}^{R}-c^{R}}{d^{R}-n_{j}^{R}}+1
$$

If $m^{R} \in \mathbb{Q}^{+}$there will be a limit on the number of independent orientations in the screw system that can be defined. If $m^{R}<m$, the orientation of the positions in the task can no longer be fully arbitrary, and $m-m^{R}$ orientations will be dependent on the arbitrary $m^{R}$ rotations. It is important to remark that this only can happen when $n_{j}^{R}<d^{R}$.

A similar counting can be done for the translational component of $S E(3)$. However, $\mathbb{R}^{3}$ is a normal subgroup of $S E(3)$ and thus the equations can not be decoupled. Following the same process as Eqn. (30) and Eqn. (33),

$$
m^{T}=\frac{n_{s}-c}{d^{T}-n_{j}}+1
$$

where $d^{T}=\operatorname{dim}\left(\mathfrak{L} \cap \mathbb{R}^{3}\right)$.

As can be seen from Eqn. (34), the translational part depends on all the parameters and variables while only providing $d^{T}$ independent equations. A serial mechanism will be solvable using only translational information if $m^{T} \in \mathbb{Q}^{+}$, which can only happen in systems with one or two single degreeof-freedom joints.

As a consequence, systems where $m^{R}<m$ and $m^{T} \notin \mathbb{Q}^{+}$will not be solvable for arbitrary positions. The positions need to be generated taking into account the limitations of the mechanism in $S O(3)$ to have a finite number of solutions.

\subsection{Defining Tasks}

When defining a task three different scenarios with $m \in \mathbb{Q}^{+}$can be identified depending on the topology of the mechanism to be synthesized.

1. When $m^{R} \geq m$ and $m \in \mathbb{Q}^{+}$the kinematic chain is fully solvable for $m$ arbitrary spatial positions. Additionally if $m^{T} \in \mathbb{Q}^{+}$, the kinematic chain can be solved for only point locations without considering orientations.

2. When $m^{R}<m$ and $m, m^{R}, m^{T} \in \mathbb{Q}^{+}$the kinematic chain can still be solved for arbitrary spatial positions. However, only $m^{R}$ positions may contain arbitrary rotations. An additional $m^{T}=\frac{d m-d^{R} m^{R}}{d}$ positions with only arbitrary translational component will have to be defined. 
3. When $m^{R}<m$ with $m, m^{R} \in \mathbb{Q}^{+}$, and $m^{T} \notin \mathbb{Q}^{+}$the rotational component will be solvable for the mechanism. However, the translational part will not have a finite number of solutions. This type of topology can not be used to perform exact kinematic synthesis for arbitrary positions. It is still possible to solve for finite solutions if the task is generated within $\mathfrak{L}$ in such a way that the rotational part is not overdetermined, yet still possible to obtain the joint parameters for each position.

\section{Tree Kinematic Synthesis}

Once a contracted tree graph representing a kinematic structure is obtained, rigid-body guidance for a finite number of task positions can be performed. For this, it is necessary to calculate the number of positions needed for all the end-effectors to fully determine the kinematic structure and thus to have a finite number of solutions.

As with serial kinematic chains, constraints are assigned to all vertices, degrees of freedom to all end-effector vertices, and joint variables and structural parameters to all edges. In the case of the degrees of freedom and joint variables, which depend on the number of relative positions $(m-1)$, only the coefficient multiplying $(m-1)$ will be represented. This will allow the usage of matrices to simplify the equations. The constraints are design parameters and thus are known.

\subsection{Design Equations}

The design equations for tree systems are stated as in Eqn. (27), applying them to all branches at the same time. This can be written as

$$
\hat{P}_{1 k}^{i}=\underbrace{\prod_{j=1}^{k_{i}} e^{\frac{\Delta \hat{\theta}_{j}^{k}}{2}} S_{j}}_{\text {common }} \underbrace{\prod_{j=k_{i}+1}^{n_{i}} e^{\frac{\Delta \hat{\theta}_{i, j}^{k}}{2} S_{i, j}}}_{\text {branch }} \begin{aligned}
& \begin{array}{l}
i=1, \ldots, b \\
k=2, \ldots, m,
\end{array} \\
& k=2, \ldots, m,
\end{aligned}
$$

where the number of common joints is indicated by $k_{i}$ and the number of end-effectors, or branches, is indicated by $b$.

The definition of the task for the design equations is similar to what is done in the previous section. Due to having multiple end-effectors, tasks become sets of displacements. In the case of tree topologies more care must 
be taken to avoid task degeneration. This is explained in detail in the next section.

\subsection{Solving Substitutions}

If the tree structure has had substitutions of parallel or serial kinematic chains, once the reduced rooted tree graph has been solved, the substitutions can be undone and the solution used to create constraints allowing the substituted chain to be solved.

The substituted serial chains has both rigid bodies on either side determined, for each position. It is then possible to consider one of the rigid bodies a reference and consider the other rigid body as the end-effector of the chain with a motion equivalent to the relative motion between both rigid bodies. However, as the substitution was done to obtain a finite number of solutions, there will be subspaces of solutions. Equation (27) can be applied to perform the synthesis of the subspace.

In the case of substituted parallel chains, each branch can be treated as a separate individual serial chain and can be solved using the same procedure used for the substituted serial chains.

\section{Matrix Representation}

This section covers the usage of some matrices associated to the tree topologies. These are used for testing for degeneration in the solvability of the exact synthesis problem.

For a tree mechanism with $v$ vertices, $e$ edges and $b$ branches, the $e \times b$ reduced end-effector path matrix $[\tilde{T}]$ is constructed by calculating the $e \times$ $(v-1)$ path matrix $[T]$ and eliminating all columns that do not correspond to an end-effector. The path matrix is defined as,

$$
[T]=\left[\begin{array}{cccc}
t_{1,1} & t_{1,2} & \cdots & t_{1, v-1} \\
t_{2,1} & t_{2,2} & \cdots & t_{2, v-1} \\
\vdots & \vdots & \ddots & \vdots \\
t_{e, 1} & t_{e, 2} & \cdots & t_{e, v-1}
\end{array}\right] \quad \text { edge } i
$$

where, 


$$
t_{i, j}=\left\{\begin{array}{l}
1 \quad \begin{array}{l}
\text { if edge } i \text { lies on the path originating at the root } \\
\text { and terminating at the vertex } j \\
0 \quad \text { otherwise. }
\end{array}
\end{array}\right.
$$

The $(v-1) \times e$ reduced incidence matrix $[\tilde{B}]$ can be constructed as,

$$
[\tilde{B}]=\left[\begin{array}{cccc}
b_{1,1} & b_{1,2} & \cdots & b_{1, e} \\
b_{2,1} & b_{2,2} & \cdots & b_{2, e} \\
\vdots & \vdots & \ddots & \vdots \\
b_{v-1,1} & b_{v-1,2} & \cdots & b_{v-1, e}
\end{array}\right] \quad \text { vertex } i
$$

where,

$$
\tilde{b}_{i, j}= \begin{cases}1 & \text { if vertex } i \text { is connected to edge } j \\ 0 & \text { otherwise }\end{cases}
$$

The matrix representation of the graph can be used to work with and define the conditions for exact kinematic synthesis. For this purpose, vectors that represent the number of joint degrees-of-freedom and structural parameters for the edges and the number of degrees-of-freedom and constraints of the nodes shall be defined.

\subsection{Task Sizing using Matrices}

In order to be able to perform exact kinematic synthesis, the global system of equations obtained using Eqn. (35) must be solvable. This in turn defines the maximum number of positions that can be required, as it is done with simple serial chains. Notice that we require the same number of positions $m$ for each end-effector. However, for exact synthesis of tree topologies, the criterion is necessary but not sufficient due to the existence of subgraphs, which will be discussed in the next subsection.

The number of unknowns for each edge are arranged into two $e \times 1$ vectors, denoted as $\mathbf{D}_{j}^{e}$ for joint degrees-of-freedom and $\mathbf{D}_{s}^{e}$ for structural parameters, so that the total number of unknowns is 


$$
n_{x}=\left((m-1) \mathbf{D}_{j}^{e}+\mathbf{D}_{s}^{e}\right)^{\mathrm{T}} \mathbf{E}
$$

where $\mathbf{E}$ is a vector of ones for the edges in the graph considered.

The number of node equations is obtained using $b \times 1$ vectors, denoted as $\mathbf{D}_{e e}^{n}$ for the end-effector degrees-of-freedom and $\mathbf{D}_{c}^{n}$ for the constraints on each branch,

$$
n_{f}=\left((m-1) \mathbf{D}_{e e}^{n}+\mathbf{D}_{c}^{n}\right)^{\mathrm{T}} \mathbf{B} .
$$

In this equation, we can take $\mathbf{B}$ as a $b \times 1$ vector of ones corresponding to branches, or end-effectors. Another option, if we want to keep the information of what constraint is associated to what vertex, is to take $\mathbf{B}$ as the vector for the vertices in the graph considered, not counting the root vertex. In this case we need to modify vectors $\mathbf{D}_{e e}^{n}$, adding zeros in those vertices that are not end-effectors, and $\mathbf{D}_{c}^{n}$, which will associate constraints to specific branches.

In order for the system to have a finite number of solutions, $n_{f}=n_{x}$ must be imposed, from which the number positions $m$ needed for exact synthesis can be obtained,

$$
m=\frac{\left(\mathbf{D}_{s}^{e}\right)^{\mathrm{T}} \mathbf{E}-\left(\mathbf{D}_{c}^{n}\right)^{\mathrm{T}} \mathbf{B}}{\left(\mathbf{D}_{e e}^{n}\right)^{\mathrm{T}} \mathbf{B}-\left(\mathbf{D}_{j}^{e}\right)^{\mathrm{T}} \mathbf{E}}+1
$$

where the system will be unsolvable if $m \notin \mathbb{Q}^{+}$. However, this criteria is necessary, but not sufficient for the system to be solvable.

Additionally, as seen in Section 6.3 for serial chains, care must be taken with the subgroups $S O(3)$ and $\mathbb{R}^{3}$ of $S E(3)$. This can be done by defining the number of positions needed for the rotational part $m^{R}$ of the mechanism as,

$$
m^{R}=\frac{\left(\mathbf{D}_{s}^{e R}\right)^{\mathrm{T}} \mathbf{E}-\left(\mathbf{D}_{c}^{n R}\right)^{\mathrm{T}} \mathbf{B}}{\left(\mathbf{D}_{e e}^{n R}\right)^{\mathrm{T}} \mathbf{B}-\left(\mathbf{D}_{j}^{e R}\right)^{\mathrm{T}} \mathbf{E}}+1
$$

where the supraindex ${ }^{R}$ denotes the restriction to the number of equations or variables that affect the $S O(3)$ component of the design equations (27) only. 
The criteria is then exactly the same as defined for serial chains in Section 6.4, although translation-only tasks have not been covered as they are of little or no interest for tree topologies due to the limitation of only one or two joints per serial chain.

\subsection{Subgraphs}

It is sometimes possible to find proper subgraphs within the tree graph representing a kinematic structure that is solvable separately. These subgraphs will generally need a different amount of end-effector positions to be solved for, and will always end up having a smaller equation system size when performing exact kinematic synthesis. However, if subgraphs exist within the system, not all end-effectors will necessarily need the same number of positions. This is because, for a given number of positions as calculated by Eqn. (42), it may so happen that a subgraph becomes overdetermined.

An interesting consequence of this phenomenon is that it only allows for simultaneous solution of tree kinematic structures where all chains from the root to end-effector have more or equal mobility than needed to reach the entire linkage locus space $\mathfrak{L}$ of the end-effector. If any kinematic chain has less mobility than needed to reach the entire linkage locus space $\mathfrak{L}$ of the endeffector, it will always form a subgraph that is solvable separately. Figure 3 presents an example of a graph with solvable subgraphs.

Hence a sufficient, but not necessary condition for the system to be solvable is that it must have no proper solvable subgraphs, that is, subgraphs that comply with $m \in \mathbb{Q}^{+}$using Eqn. (42). In order to check for this condition, all possible proper subgraphs must be tested. This can be expressed using the reduced path matrix,

$$
\mathbf{E}_{i}=\left.[\tilde{T}] \tilde{\mathbf{B}}_{i}^{e e}\right|_{[>0]}
$$

where $\tilde{\mathbf{B}}_{i}^{e e}$ is a $b \times 1$ vector representing the end-effectors considered for a given subgraph $i$. The Iverson bracket $[>0]$ is used to denote that elements of $\mathbf{E}$ vector should be either be 1 if $>0$ or 0 otherwise. There are $2^{b}-2$ possible subgraphs for any given rooted tree graph representing a reduced kinematic structure, excluding both the full graph and the null graph.

Given $\mathbf{E}_{i}$ corresponding to the edges in a subgraph, the $\mathbf{B}_{i}$ nodes in the subgraph can be calculated by, 


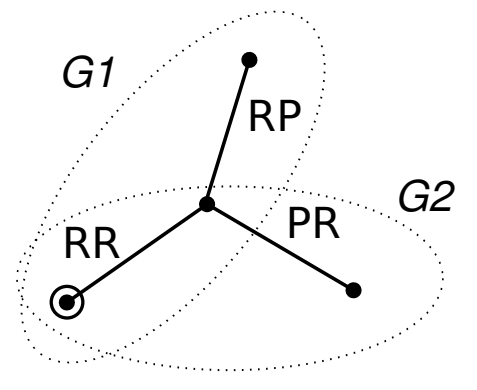

Figure 3: Both branches with fewer than $\operatorname{dim}(\mathfrak{L})=6$ joints form subgraphs G1 and G2 that are solvable separately.

$$
\mathbf{B}_{i}=\left.[\tilde{B}] \mathbf{E}_{i}\right|_{[>0]}
$$

This criteria is sufficient, but not necessary. While it does always generate the smallest system size for performing exact synthesis, it increases the number of end-effector positions needed. A more lax criteria can be used instead, in which cases where the subgraphs are separately solvable are considered.

For each subgraph $i$, Eqn. (42) can be used to calculate the number of positions needed for exact synthesis of the subgraph,

$$
m_{i}=\frac{\left(\mathbf{D}_{s}^{e}\right)^{\mathrm{T}} \mathbf{E}_{i}-\left(\mathbf{D}_{c}^{n}\right)^{\mathrm{T}} \mathbf{B}_{i}}{\left(\mathbf{D}_{e e}^{n}\right)^{\mathrm{T}} \mathbf{B}_{i}-\left(\mathbf{D}_{j}^{e}\right)^{\mathrm{T}} \mathbf{E}_{i}}+1
$$

where again we could use $\tilde{\mathbf{B}}_{i}^{e e}$ instead of $\mathbf{B}_{i}$ if we lump together all the constraints for each given branch.

A necessary and sufficient criteria for determining the solvability of a graph is, given the number of positions $m$ needed for exact synthesis of the subgraph, for all proper subgraphs $i$ in the graph

$$
m \leq m_{i}, \text { if } m_{i} \in \mathbb{Q}^{+}
$$

Additionally, as in the general case, $S O(3)$ and $\mathbb{R}^{3}$ must be considered separately. Given a number of rotational components of the $m$ positions, denoted as $m^{R}$, the following additional criteria are obtained: 


$$
m^{R} \leq m_{i}^{R}, \text { if } m_{i}^{R} \in \mathbb{Q}^{+}
$$

where $m_{i}^{R}$ are the number of frames needed to solve only the rotation part for subgraph $i$ of the mechanism.

If these criteria are not met, then the subgraph $i$ where either $m>m_{i}$ or $m^{R}>m_{i}^{R}$ becomes overdetermined, while other kinematic chains become underdetermined by the overall set of positions $m$. The system of equations for exact kinematic synthesis is no longer guaranteed to have any solutions when the same number of task positions is defined for each end-effector. In this case, the system will not be solvable.

Finally, it must be noted that checking a single rooted tree graph is not enough. In order to ensure that the mechanism is solvable, all the endeffectors must be set as the root node and all the subgraphs from these rooted tree graphs must be analyzed. This means that in general, in order to establish if a topology is solvable, all the $b\left(2^{b}-2\right)$ subgraphs arising from all the different possible root node arrangements must be checked.

\subsection{Solvable Tree Graph}

Definition Let $\mathrm{T}$ be a contracted tree graph representing a kinematic structure and $\mathrm{S}$ be the set of subgraphs of all possible rooted tree graphs arising from setting each end-effector of $\mathrm{T}$ as the root of the graph. $\mathrm{T}$ will be called solvable for a finite number of independent positions if,

1. $m \in \mathbb{Q}^{+}$

2. $m \leq m_{i}, \forall m_{i} \in \mathbb{Q}^{+}, i \in \mathrm{S}$

3. $m^{R} \leq m_{i}^{R}, \forall m_{i}^{R} \in \mathbb{Q}^{+}, i \in \mathrm{S}$

The advantage of solving a tree with subgraphs is that the workspace can be defined by a smaller number of positions of all end-effectors at the same time. However, the dimension of the equation system will always be larger than solving the smallest subgraph first and using the results to solve other subgraphs and eventually the entire graph.

\section{Examples}

In this section three different examples that focus on different parts of the dimensional synthesis are presented. For the notation of contracted rooted 


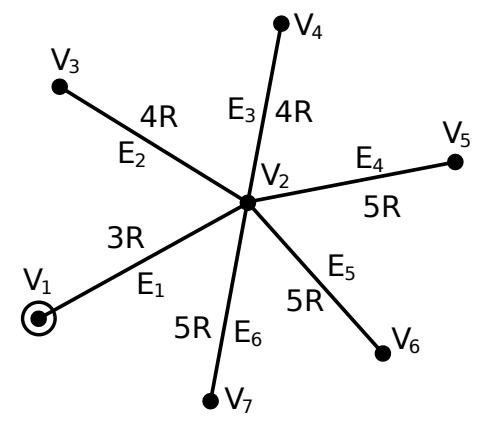

Figure 4: Rooted tree graph of an anthropomorphic hand model.

kinematic trees, hyphens will be used to indicate serial links and parenthesis to indicate parallel links.

The first example is a $3 \mathrm{R}-(4 \mathrm{R}, 4 \mathrm{R}, 5 \mathrm{R}, 5 \mathrm{R}, 5 \mathrm{R})$ manipulator, representing a human hand, where the focus is on using the matrix approach presented to determine the independently solvable subgraphs of the mechanism. The second example is a more simple RP-(R,P) mechanism where the focus is on solving the dimensional synthesis problem algebraically using the technique of changing the root node in order to make it a more tractable problem. The last example is a $2 \mathrm{R}-(2 \mathrm{R}, \mathrm{R}, \mathrm{R})$ manipulator which would be of a complexity between the other two examples, for which numeric results of a solution are presented.

\subsection{Anthropomorphic Hand Model Subgraphs}

Previously presented work performed kinematic synthesis on an anthropomorphic hand model with 5 fingers and a total of 26 revolute joints [39] and it is the first mention of dimensional kinematic synthesis applied to tree topologies. The anthropomorphic hand model will be revisited using the new tools presented in this paper for dimensional synthesis.

The anthropomorphic hand model is organized as a $3 \mathrm{R}-(4 \mathrm{R}, 4 \mathrm{R}, 5 \mathrm{R}, 5 \mathrm{R}, 5 \mathrm{R})$ tree, where the wrist is considered to have 3 revolute joints which are common to the five fingers. The index and middle fingers have 4 revolute joints, while the third finger, fourth finger and thumb have 5 revolute joints. This gives a total of 26 revolute joints. There are not any constraints besides the implicit constraints of the joints.

The contraction of the individual joints in this case is trivial as there are no closed loops and all serial chains are formed by revolute joints. The 
contracted graph can be seen in Fig. 4 and has all the edges and vertices labeled. It is also easy to see that in this case there is no edge with 6 or more revolute joints, which is a necessary, but not a sufficient condition for the structure to be solvable if there are no additional constraints.

As the system is formed entirely by revolute joints, it is straightforward to assign the $6 \times 1$ vectors associated with the joint degrees-of-freedom $\mathbf{D}_{j}^{e}$ and the structural parameters $\mathbf{D}_{s}^{e}$,

$$
\begin{aligned}
\mathbf{D}_{j}^{e} & =\left[\begin{array}{llllll}
3 & 4 & 4 & 5 & 5 & 5
\end{array}\right]^{\mathrm{T}} \\
\mathbf{D}_{s}^{e} & =\left[\begin{array}{llllll}
12 & 16 & 16 & 20 & 20 & 20
\end{array}\right]^{\mathrm{T}},
\end{aligned}
$$

as revolute joints have 4 structural parameters associated to them.

The same can be done with the vertices, which are $5 \times 1$ vectors associated to the end-effector degrees-of-freedom $\mathbf{D}_{e e}^{n}$ and constraints $\mathbf{D}_{c}^{n}$,

$$
\begin{aligned}
\mathbf{D}_{e e}^{n} & =\left[\begin{array}{lllll}
6 & 6 & 6 & 6 & 6
\end{array}\right]^{\mathrm{T}} \\
\mathbf{D}_{c}^{n} & =\left[\begin{array}{lllll}
0 & 0 & 0 & 0 & 0
\end{array}\right]^{\mathrm{T}}
\end{aligned}
$$

Notice that in this case all end-effectors have a linkage locus space $\mathfrak{L}=\mathfrak{s e}(3)$ and no additional constraints.

The number of positions needed for the entire system to perform exact kinematic synthesis can be now be computed. This is done by using Eqn. (42) with all the edges and branches,

$$
\begin{aligned}
& \mathbf{E}=\left[\begin{array}{llllll}
1 & 1 & 1 & 1 & 1 & 1
\end{array}\right] \\
& \mathbf{B}=\left[\begin{array}{lllll}
1 & 1 & 1 & 1 & 1
\end{array}\right] \\
& m=\frac{26 \cdot 4-0}{6 \cdot 5-26}+1=27
\end{aligned}
$$

Additionally the rotational components must also be checked by calculating the system that interacts with $S O(3)$, 


$$
\begin{aligned}
\mathbf{D}_{j}^{e R} & =\left[\begin{array}{llllll}
3 & 4 & 4 & 5 & 5 & 5
\end{array}\right]^{\mathrm{T}} \\
\mathbf{D}_{s}^{e R} & =\left[\begin{array}{llllll}
6 & 8 & 8 & 10 & 10 & 10
\end{array}\right]^{\mathrm{T}} \\
\mathbf{D}_{e e}^{n R} & =\left[\begin{array}{lllll}
3 & 3 & 3 & 3 & 3
\end{array}\right]^{\mathrm{T}} \\
\mathbf{D}_{c}^{n R} & =\left[\begin{array}{lllll}
0 & 0 & 0 & 0 & 0
\end{array}\right]^{\mathrm{T}}
\end{aligned}
$$

Now by using Eqn. (43) the number of rotation positions $m^{R}$ needed, considering only the rotational component, can computed as,

$$
m^{R}=\frac{26 \cdot 2-0}{3 \cdot 5-26}+1=-\frac{41}{11}
$$

With $m=27 \in \mathbb{Q}^{+}$and $m^{R}=\frac{-41}{11} \notin \mathbb{Q}^{+}$it can be seen that the system meets another necessary, but not sufficient condition of solvability. For it to be solvable all the subgraphs must comply with Eqn. (47). This can be done by first determining the reduced path matrix $[\tilde{T}]$ and reduced incidence matrix $[\tilde{B}]$. The path matrix consists on the edges that lie on the path to a given node. This is relative to the root node, which for this example is $V_{1}$. The reduced path matrix can be written as,

$$
[\tilde{T}]=\begin{gathered}
V_{3} \\
E_{1} \\
E_{2} \\
E_{3} \\
E_{4} \\
E_{5} \\
E_{6}
\end{gathered}\left[\begin{array}{ccccc}
1 & 1 & 1 & 1 & 1 \\
1 & 0 & 0 & 0 & 0 \\
0 & 1 & 0 & 0 & 0 \\
0 & 0 & 1 & 0 & 0 \\
0 & 0 & 0 & 1 & 0 \\
0 & 0 & 0 & 0 & 1
\end{array}\right]
$$

where it can be seen that there are no columns for either $V_{1}$ or $V_{2}$ as they are not end-effectors and have been eliminated.

The reduced incidence matrix $[\tilde{B}]$ is, 


$$
[\tilde{B}]=V_{4} V_{V_{3}}\left[\begin{array}{cccccc}
E_{1} & E_{2} & E_{3} & E_{4} & E_{5} & E_{6} \\
V_{5} \\
V_{6} \\
V_{7}
\end{array}\left[\begin{array}{cccccc}
1 & 1 & 1 & 1 & 1 & 1 \\
0 & 1 & 0 & 0 & 0 & 0 \\
0 & 0 & 1 & 0 & 0 & 0 \\
0 & 0 & 0 & 1 & 0 & 0 \\
0 & 0 & 0 & 0 & 1 & 0 \\
0 & 0 & 0 & 0 & 0 & 1
\end{array}\right]\right.
$$

where it can be seen that there is no first row for the root vertex $V_{1}$.

Now the end-effectors must be iterated on using Eqn. (44) to construct the edges leading to the end-effectors and Eqn. (45). There are in general $2^{b}-2$ possible proper subgraphs given a root node. However, in this case there are symmetries as many branches are the same leading to many equivalent subgraphs. For single branches they can be studied using the single serial chain equation from Eqn. (30) and in this case it is easy to see that all branches need a negative number positions. Therefore none of these subgraphs are solvable systems.

Next the two branches with 4 revolute joints (index and middle finger) are considered,

$$
\tilde{\mathbf{B}}_{6}^{e e}=\left[\begin{array}{lllll}
1 & 1 & 0 & 0 & 0
\end{array}\right]^{\mathrm{T}}
$$

The system has the following edges,

$$
\begin{aligned}
\mathbf{E}_{6}=\left.[\tilde{T}] \tilde{\mathbf{B}}_{6}^{e e}\right|_{[>0]} & =\left.\left[\begin{array}{llllll}
2 & 1 & 1 & 0 & 0 & 0
\end{array}\right]^{\mathrm{T}}\right|_{[>0]} \\
& =\left[\begin{array}{llllll}
1 & 1 & 1 & 0 & 0 & 0
\end{array}\right]^{\mathrm{T}}
\end{aligned}
$$

and the following vertices,

$$
\mathbf{B}_{6}=[\tilde{B}] \mathbf{E}_{6}=\left[\begin{array}{lllllll}
1 & 1 & 1 & 1 & 0 & 0 & 0
\end{array}\right]^{\mathrm{T}}
$$

It can also be seen graphically in Fig. 4 that this system has indeed only the edges $E_{1}, E_{2}, E_{3}$ and vertices $V_{1}, V_{2}, V_{3}, V_{4}$. The value $m_{6}$ can be calculated by Eqn. (46), 
Table 3: All solvable subgraphs of the anthropomorphic hand model

\begin{tabular}{ccccc}
\hline Subgraph & $b$ & $r$ & $m$ & $n_{f}=n_{x}$ \\
\hline 3R-(4R,4R,5R,5R,5R) & 5 & 26 & 27 & 780 \\
3R-(4R,5R,5R,5R) & 4 & 22 & 45 & 1056 \\
3R-(4R,4R,5R,5R) & 4 & 21 & 29 & 672 \\
3R-(4R,5R,5R) & 3 & 17 & 69 & 1224 \\
3R-(4R,4R,5R) & 3 & 16 & 33 & 576 \\
3R-(4R,4R) & 2 & 11 & 45 & 528 \\
\hline
\end{tabular}

$$
m_{6}=\frac{4 \cdot 11-0}{6 \cdot 2-11}+1=45
$$

Furthermore $m_{6}^{R}$ can be calculated as,

$$
m_{6}=\frac{2 \cdot 11-0}{3 \cdot 2-11}+1=-\frac{17}{5}
$$

With $m_{6}^{R} \notin \mathbb{Q}^{+}$and $m_{6}=45 \geq m=27$ this subgraph is solvable. This process has to be continued for all the possible subgraphs of this rooted tree graph and other rooted tree graphs arising from setting the root to the other end-effectors $\left\{V_{3}, V_{4}, V_{5}, V_{6}, V_{7}\right\}$. However, for this mechanism, there exists no separately solvable subgraph that is not already a subgraph of the original rooted tree graph. If all these possible subgraphs meet the criteria it can be said that the graph is solvable.

After analyzing all the possible subgraphs, the results seen in Table 3 can be obtained. From this it can be seen that all the possible subgraphs were found in [39] and that the entire system is solvable. It is also important to note that all the end-effectors have $\mathfrak{L}=\mathfrak{s e}(3)$ and $\mathfrak{L} \cap \mathfrak{s o}(3)=\mathfrak{s o}(3)$. Therefore all $m$ positions defined in the task can have arbitrary orientation and location. This is generally true for all unconstrained pure revolute joint systems that are solvable.

Now that the task specifications have been defined for the mechanism, synthesis can be performed for a specific task, consisting of $m$ spatial positions, by solving the design equations from Eqn. (35). In our previous work 


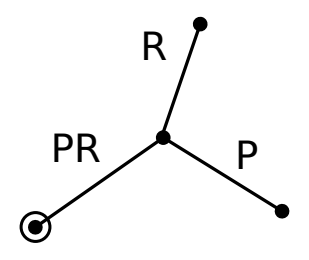

Figure 5: Rooted tree graph of the RP-(R,P) mechanism.

[39, 45] a numerical optimization scheme has been employed, based on a genetic algorithm and a Levenberg-Marquadt minimization, which has been proven to be successful at finding operative solutions.

\subsection{Algebraic $P R-(R, P)$}

The PR-(R,P) consists of two common joints: one prismatic and one revolute, which fork into two branches, one being a revolute joint and the other a prismatic joint as seen in Fig. 5. In this case, there are two subgraphs corresponding to a PRR mechanism and a PRP mechanism. To decrease the global equation system size, either subgraph can be solved individually and then the results can be used to solve the remaining joint. However, this requires more task positions for the first branch solved than for the remaining joint. Heterogeneous numbers of positions for each end-effector are generally not desired.

From Eqn.(42) the number of positions needed for all possible chains can be counted as shown in Table 2, The PRR chain needs $m=3$ positions, the PRP chain needs $m=3 \frac{1}{2}$ positions and the entire system needs $m=2 \frac{1}{2}$ positions. Notice that the entire system needs fewer positions than either branch.

It can be seen, using Eqn. (43), that the PRR chain needs $m^{R}=5$ positions, the PRP chain needs $m^{R}=2$ position and the entire system would also need $m^{R}=2$ positions. In the case of the PRP chain, the exact synthesis is limited by $m^{R}$ to only two positions with a rotational component. Therefore when defining the task, some additional positions without orientation information will have to be added.

In order to make sure the system is still solvable, the limit of translations $m^{T}$ must also be checked for all subgraphs. For the PRR and PRP chain $m^{T}=\infty$ is obtained, while for the entire system $m^{T}=7$ is obtained. This indicates that only the entire system is solvable for only translations 
Table 4: Solvability of the different subgraphs of the PR-(R,P) mechanism

\begin{tabular}{|c|c|c|c|c|}
\hline Subgraph & $m$ & $m^{R}$ & $m^{T}$ & Comments \\
\hline $\mathrm{PR}-(\mathrm{R}, \mathrm{P})$ & $2 \frac{1}{2}$ & 2 & 7 & $\begin{array}{l}\text { Full system, rotational and translational com- } \\
\text { ponents solvable }\end{array}$ \\
\hline PRR & 3 & 5 & $\infty$ & Full system and rotational component solvable \\
\hline PRP & $3 \frac{1}{2}$ & 2 & $\infty$ & $\begin{array}{l}\text { Only rotational component solvable } m^{R}<m \\
\text { and } m^{T} \notin \mathbb{Q}^{+}\end{array}$ \\
\hline
\end{tabular}

and additionally that the PRP subgraph is only solvable using rotational components. The results of the solvability are summed up in Table 4.

Being a simple mechanism it can also be solved algebraically. Consider the kinematic chain from one end-effector to another. This can be thought of as changing the root node to one of the end effectors and then solving a subgraph. The initial forward kinematics of both branches can be written as,

$$
\begin{aligned}
& \hat{S}_{P}^{c}\left(d^{c}\right) \hat{S}_{R}^{c}\left(\theta^{c}\right) \hat{S}_{R}(\theta)=\hat{P}_{P R R} \\
& \underbrace{\hat{S}_{P}^{c}\left(d^{c}\right) \hat{S}_{R}^{c}\left(\theta^{c}\right)}_{\text {common }} \underbrace{\hat{S}_{P}(d)}_{\text {branch }}=\underbrace{\hat{P}_{P R P}}_{\text {pose }}
\end{aligned}
$$

By considering the kinematic chain from one end-effector to another the common joints can be eliminated leading to the following kinematic chain:

$$
\hat{S}_{P}(d)^{-1} \hat{S}_{R}(\theta)=\hat{P}_{P R P}^{-1} \hat{P}_{P R R}
$$

where $\hat{S}_{P}(d)^{-1}$ is equivalent to $\hat{S}_{P}(-d)$. This is analogous to making the end-effector at the end of the $\mathrm{P}$ edge the root of the system and solving the subgraph formed by the PR branch in the new tree graph.

This can be solved algebraically and gives a single solution. Given the solution, the position of the rigid body connecting the common branches can be solved as a serial chain,

$$
\hat{S}_{P}^{c}\left(d^{c}\right) \hat{S}_{R}^{c}\left(\theta^{c}\right)=\hat{P}_{P R P} \hat{S}_{P}(-d)
$$


which also has a single solution. Furthermore the entire system has a single solution.

Therefore the problem can be treated as two separate PR chains and solved individually. The number of positions each PR chain needs is $m=2 \frac{1}{2}$ and is coincidentally the same as the number of positions needed for the entire system. The solution process is the same as in [46].

Having $m=2 \frac{1}{2} \notin \mathbb{N}^{+}$, fully arbitrary positions cannot be defined. With $m^{R}=2$ and $m^{T}=7$, the translation of three positions and the orientation of two of them can be defined in order to perform kinematic synthesis. If more than 2 orientations are defined, the rotational component of the system becomes overdetermined. The positions can be defined as,

$$
\begin{aligned}
& \hat{P}_{12}=\left.\hat{P}_{P R P}^{-1} \hat{P}_{P R R}\right|_{12}=s_{12}^{w}+\mathbf{b}_{12}+\epsilon\left(\mathrm{s}_{12}^{w 0}+\mathbf{b}_{12}^{0}\right) \\
& \hat{P}_{13}=\left.\hat{P}_{P R P}^{-1} \hat{P}_{P R R}\right|_{13}=s_{12}^{w}+\mathbf{b}_{12}+\epsilon\left(\mathrm{s}_{13}^{w 0}+\mathbf{b}_{13}^{0}\right)
\end{aligned}
$$

where it can be seen that they share the same orientation.

Using the real part, the rotation axis of the $\mathrm{R}$ joint, $\mathrm{g}$, and the rotation angle about it, $\boldsymbol{\theta}$, can be computed as,

$$
\mathbf{g}=\frac{\mathbf{b}_{12}}{\left\|\mathbf{b}_{12}\right\|}, \quad \tan \boldsymbol{\theta}=\frac{\left\|\mathbf{b}_{1 i}\right\|}{s_{1 i}^{w}}, \quad i=2,3
$$

The dual part can then be used to solve for the moment of the line defining the $\mathrm{R}$ joint, $\mathrm{g}^{0}$, and for the direction of the $\mathrm{P}$ joint, $\mathbf{h}$,

$$
\mathbf{g}^{0}=\mathbf{b}_{1 i}^{0}-\frac{d_{1 i}}{2}\left(\cos \frac{\theta_{1 i}}{2} \mathbf{h}+\sin \frac{\theta_{1 i}}{2} \mathbf{g} \times \mathbf{h}\right), \quad i=2,3
$$

by imposing $\|\mathbf{h}\|=1$ and

$$
\frac{s_{12}^{w 0}}{\frac{d_{12}}{2} \sin \frac{\theta_{12}}{2}}=\frac{s_{13}^{w 0}}{\frac{d_{13}}{2} \sin \frac{\theta_{13}}{2}}
$$

This characterizes the RP joint formed by the two end-effector edges. Knowing the slide and direction of the $\mathrm{P}$ joint, $d_{1 i}$ and $\mathbf{h}$, the relative transformation from the end-effector of the $\mathrm{P}$ joint to the vertex between the PR 


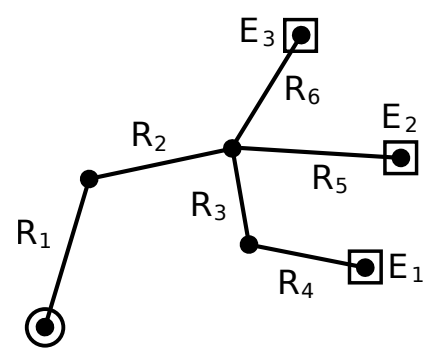

Figure 6: Rooted tree graph of the RR-(RR,R,R) mechanism. In order to more easily identify the individual joints, this graph is displayed without being compacted.

chain and the $\mathrm{P}$ joint can be calculated to obtain two new relative transformations,

$$
\begin{aligned}
& \hat{S}_{12}^{c}=\left.\hat{P}_{P R P} \hat{S}_{P}(-d)\right|_{12}=s_{12}^{w c}+\mathbf{b}_{12}^{c}+\epsilon\left(\mathrm{s}_{12}^{w 0 c}+\mathbf{b}_{12}^{0 c}\right) \\
& \hat{S}_{13}^{c}=\left.\hat{P}_{P R P} \hat{S}_{P}(-d)\right|_{13}=s_{12}^{w c}+\mathbf{b}_{12}^{c}+\epsilon\left(\mathrm{s}_{13}^{w 0 c}+\mathbf{b}_{13}^{0 c}\right)
\end{aligned}
$$

The procedure follows as before where the new system is determined by using Eqn. (65) and Eqn. (66), and thus obtaining all the joint variables and structural parameters of the system.

The entire system yields a single unique solution. The method for solving the RP-(R,P) kinematic structure is not general as not all kinematic structures can be split into two non-overlapping substructures that can be solved with the same number of task positions. However, this example shows a novel methodology that can be used on many structures.

\subsection{Numeric $R R-(R R, R, R)$}

The RR-(RR,R,R) can be thought of as a very simple hand model. The graph of the mechanism can be seen in Fig. 6. It has two revolute joints in the wrist $\left(R_{1}\right.$ and $R_{2}$ ), two fingers with a single revolute joint each for holding objects $\left(R_{5}\right.$ and $\left.R_{6}\right)$ and a thumb with two revolute joints in order to perform more dexterous object manipulation $\left(R_{3}\right.$ and $\left.R_{4}\right)$.

As with any other tree, the first step is to identify all the subgraphs and determine if it is possible to perform exact dimensional synthesis on the mechanism. For the sake of brevity this is not analyzed in depth for this 
Table 5: Input task positions for the $2 \mathrm{R}-(2 \mathrm{R}, \mathrm{R}, \mathrm{R})$ mechanism given as dual quaternions

\begin{tabular}{rr|l}
\hline \multicolumn{2}{c|}{ Frame } & $\hat{P}$ \\
\hline$E_{1}$ & 1 & $0.368-0.737 i-0.446 j-0.350 k+\epsilon(0.174 i+3.098 j-0.493 k+3.635)$ \\
& 2 & $0.113+0.835 i+0.402 j+0.359 k+\epsilon(6.005 i-6.954 j-4.810 k-4.340)$ \\
& 3 & $0.759-0.638 i-0.109 j-0.073 k+\epsilon(-0.023 i+4.115 j+2.019 k+0.764)$ \\
\hline & 1 & $0.070+0.621 i+0.665 j+0.409 k+\epsilon(2.076 i-0.232 j-2.086 k-4.013)$ \\
$E_{2}$ & 2 & $0.967-0.027 i-0.191 j-0.165 k+\epsilon(1.306 i+1.974 j+1.998 k+0.769)$ \\
& 3 & $0.998+0.002 i+0.063 j+0.014 k+\epsilon(-0.169 i+1.106 j-0.325 k-0.065)$ \\
\hline & 1 & $0.356+0.392 i+0.678 j+0.510 k+\epsilon(-4.285 i+4.559 j-1.975 k-1.126)$ \\
$E_{3}$ & 2 & $0.975-0.005 i-0.214 j-0.065 k+\epsilon(0.811 i+0.932 j+0.588 k+0.248)$ \\
& 3 & $0.614+0.619 i+0.487 j+0.054 k+\epsilon(0.129 i+0.586 j-3.281 k-0.307)$ \\
\hline
\end{tabular}

mechanism, although it can be seen that it is indeed solvable for $m=3$ positions.

Consider a randomly generated task consisting of three positions for each end-effector, as shown in Table 5. Equation (35) is then solved numerically using a global optimization algorithm based on the fusion of a Genetic Algorithm and Levenberg-Marquadt local optimizer [39]. Many different solutions are obtained, making it necessary to establish some criteria to chose the desired solution.

The numerical results for one of the solutions are shown in Table6, including screw axes and inverse kinematics for the defined task. See an example of implementation of the presented solution in Fig. 7.

\section{Conclusions}

This paper presents a methodology for the finite-position dimensional synthesis of articulated systems with a tree structure. The design process is based on existing techniques for the dimensional synthesis of spatial serial chains; however, in the design of tree-like topologies specific issues appear that are explored and solved for the first time in this work.

The method is based on representing the tree as a rooted graph and performing reduction operations in order to simplify the initial topology. 
Table 6: An example solution for the RR-(RR,R,R) mechanism. The joints are described using the Plücker coordinates in the reference frame

\begin{tabular}{c|cccc}
\hline & $\mathbf{s}$ & $\mathbf{s}_{0}$ & $\Delta \theta^{2}$ & $\Delta \theta^{3}$ \\
\hline$R_{1}$ & $(0.856,0.376,0.354)$ & $(-3.292,3.415,4.339)$ & 0.383 & 4.142 \\
$R_{2}$ & $(-0.497,-0.473,-0.728)$ & $(-1.510,-8.052,6.262)$ & 1.000 & 1.883 \\
$R_{3}$ & $(-0.870,-0.355,0.341)$ & $(-26.900,51.540,-15.030)$ & 0.532 & 0.290 \\
$R_{4}$ & $(-0.300,-0.705,-0.642)$ & $(8.196,0.882,-4.798)$ & 6.401 & 1.226 \\
$R_{5}$ & $(-0.505,-0.698,-0.508)$ & $(-6.383,1.045,4.905)$ & 2.842 & 5.358 \\
$R_{6}$ & $(0.155,0.772,0.616)$ & $(0.055,1.364,-1.724)$ & -2.194 & 3.167 \\
\hline
\end{tabular}

The forward kinematics equations for each of the serial chains forming the tree articulated system are stated and equated to a set of task positions, from which conditions for the solvability of the whole tree and individual subgraphs or branches of the tree are derived. The inclusion of tree articulated systems among possible candidate topologies for the design process allows to extend dimensional synthesis to systems with a high number of degrees-of-freedom and more complex motion tasks.

This methodology's main application is the design of novel multi-fingered hands for human-robot interaction, where a non-anthropomorphic hand could be used to perform tasks in cooperation with humans. The method is currently limited to the exact finite-position synthesis and hence it does not account for performance issues derived from the pass from a finite set of positions to a continuous trajectory, such as the existence of self-intersections or singularities while performing the task. The addition of conditions for these and other performance requirements, as well as for grasping, is to be considered in future work.

\section{Acknowledgements}

This work is supported by the National Science Foundation under Grant No. 1208385. The content is solely the author's responsibility. 

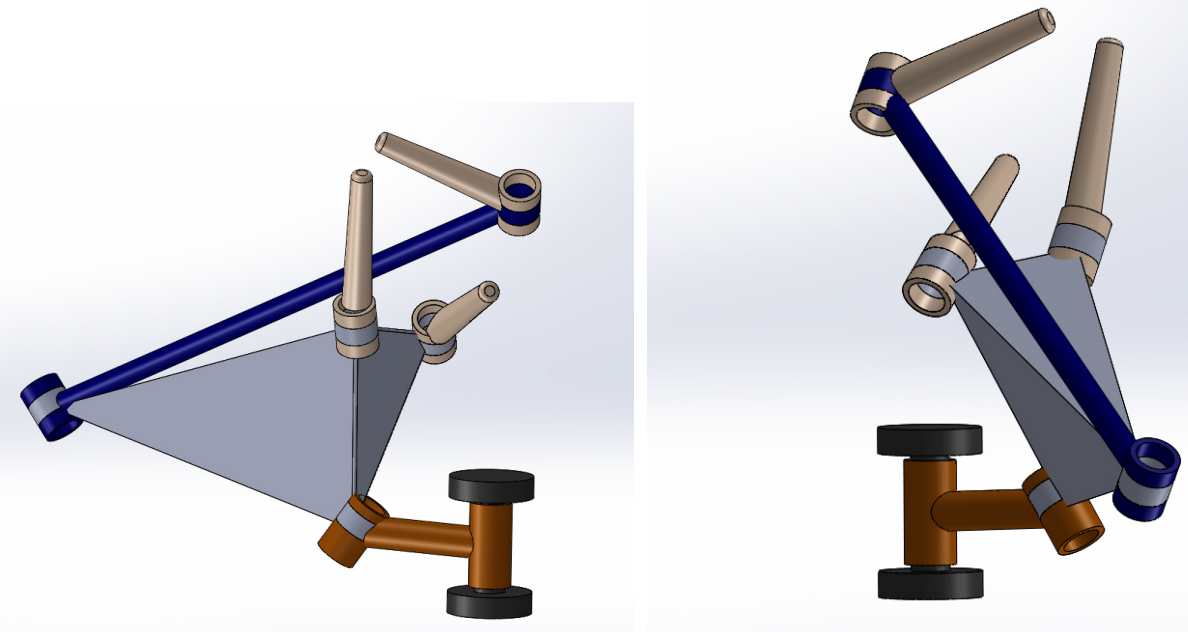

Figure 7: Two views of a possible implementation for the selected solution.

\section{References}

[1] R. Hartenberg, J. Denavit, Kinematic Synthesis of Linkages, McGrawHill, 1964.

[2] B. Roth, Finite position theory applied to mechanism synthesis, Journal of Applied Mechanics 34 (1967) 599-605.

[3] G. N. Sandor, A. G. Erdman, Advanced Mechanism Design: Analysis and Synthesis, Vol. 2, Prentice-Hall, Englewood Cliffs, NJ, 1984.

[4] C. Suh, C. Radcliffe, Kinematics and Mechanisms Design, John Wiley, 1978.

[5] P. Chen, B. Roth, Design equations for finitely and infinitesimally separated position synthesis of binary link and combined link chains, ASME Journal of Engineering for Industry 91 (1967) 209-219.

[6] G. Sandor, K. Bishopp, On a general method of spatial kinematic synthesis by means of a stretch-rotation tensor', Journal of Engineering for Industry 91 (1969) 115-122. 
[7] E. Lee, C. Mavroidis, Geometric design of 3r manipulators for reaching four end-effector spatial poses, The International Journal of Robotics Research 23(3) (2004) 247-254.

[8] J. M. McCarthy, G. Soh, Geometric Design of Linkages, Springer-Verlag, New York, second edition, 2010.

[9] M. L. Husty, M. Pfurner, H.-P. Schrocker, K. Brunnthaler, Algebraic methods in mechanism analysis and synthesis, Robotica 25 (2007) 661675.

[10] J. Angeles, Spatial Kinematic Chains. Analysis, Synthesis, Optimization, Springer-Verlag, New York, 1982.

[11] J. Merlet, D. Daney, Dimensional synthesis of parallel robots with a guaranteed given accuracy over a specific worskpace, in: Proc. of the 2005 International Conference on Robotics and Automation, Barcelona, Spain, pp. 942-947.

[12] H. Su, J. M. McCarthy, L. T. Watson, Generalized linear product homotopy algorithms and the computation of reachable surfaces, ASME Journal of Computers and Information Science and Engineering 4(3) (2004) 226-235.

[13] G. Gogu, Structural Synthesis of Parallel Robots. Part 1: Methodology, Springer, first edition, 2007.

[14] J. M. Herve, The lie group of rigid body displacements, a fundamental tool for mechanism design, Mechanism and Machine Theory 34 (1999) $717-730$.

[15] L. Woo, Type synthesis of plane linkages, ASME Journal of Engineering for Industry (1967) 159-172.

[16] M. Huang, A. Soni, Application of linear and nonlinear graphs in structural synthesis of kinematic chains, Journal of Engineering for Industry (1973) 525-532.

[17] N. Manolescu, A method based on baranov trusses and using graph theory to find the set of plane jointed kinematic chains and mechanisms, Mechanisms and Machine Theory 8 (1977) 3-22. 
[18] F. Freudenstein, E. R. Maki, The creation of mechanisms according to kinematic structure and function, Journal of Environment and Planning 6 (1979) 375-391.

[19] L. W. Tsai, Mechanism Design: Enumeration of Kinematic Structures According to Function, CRC Press, Boca Raton, 2001.

[20] T. Mruthyunjaya, Kinematic structure of mechanisms revisited, Mechanism and Machine Theory 38 (2003) 279-320.

[21] Y. Lu, e. a. Mao, B.Y., Derivation of valid contracted graphs from simpler contracted graphs for type synthesis of closed mechanisms, Mechanism and Machine Theory 52(3) (2012) 206-218.

[22] Y. Lu, e. a. Mao, B.Y., Analysis and determination of associated linkage, redundant constraint, and degree of freedom of closed mechanisms with redundant constraints and/or passive degree of freedom, ASME Journal of Mechanical Design 134(5) (2012).

[23] C. Chuang, J. Lee, Topological synthesis of underactuated passively adaptive finger mechanisms, in: Proc. of the 13th World Congress in Mechanism and Machine Science, Guanajuato, Mexico.

[24] J. M. Selig, Geometric Fundamentals of Robotics (Monographs in Computer Science), SpringerVerlag, 2004.

[25] N. Biggs, Discrete Mathematics, 1985.

[26] I. Chen, G. Yang, I. Kang, Numerical inverse kinematics for modular reconfigurable robtos, Journal of Robotic Systems 16(4) (1999) 213-225.

[27] G. Song, N. Amato, A motion-planning approach to folding: from paper craft to protein folding, IEEE Transactions on Robotics and Automation 20(1) (2004) 60-71.

[28] A. Jain, Graph-theory roots of spatial operators for kinematics and dynamics, in: Proc. of the 2010 International Conference on Robotics and Automation, Anchorage, Alaska, USA, pp. 2745-2750.

[29] J. Garcia de Jalon, E. Bayo, Kinematic and Dynamic Simulation of Multibody Systems: The Real-Time challenge, Springer-Verlag, 1994. 
[30] S. Stramigioli, Modeling and IPC control of interactive mechanical systems - A coordinate-free approach, volume LNCIS 266, Springer, 2001.

[31] C. Tischler, A. Samuel, K. Hunt, Kinematic chains for robot hands - 1. orderly number synthesis, Mechanism and Machine Theory 30(8) (1995) $1193-1215$.

[32] C. Tischler, A. Samuel, K. Hunt, Kinematic chains for robot hands ii: Kinematic constraints, classification, connectivity and actuation, Mechanism and Machine Theory 30(8) (1995) 1217-1239.

[33] L. Briglen, T. Laliberte, C. Gosselin, Underactuated Robotic Hands, Springer, 2008.

[34] A. Ciocarlie, P. Allen, Data-driven optimization for underactuated robotic hands, in: Proc. of the 2010 International Conference on Robotics and Automation, Anchorage, Alaska, USA, pp. 1292-1299.

[35] J. Belter, A. Dollar, Performance characteristics of anthropomorphic prosthetic hands, in: Proceedings of the 2011 IEEE International Conference on Rehabilitation Robotics, pp. 921-927.

[36] L. Biagiotti, F. Lotti, C. Melchiorri, G. Vassura, How Far is the Human Hand? A Review of Anthropomorphic Robotic End-effectors. Internal report, Technical Report, Universita di Bologna, 2004.

[37] J. Dai, D. Wang, Geometric analysis and synthesis of the metamorphic robotic hand, ASME Journal of Mechanical Design 129 (2007) 11911197.

[38] A. Perez Gracia, J. M. McCarthy, The kinematic synthesis of spatial serial chains using clifford algebra exponentials, Proceedings of the Institution of Mechanical Engineers, Part C, Journal of Mechanical Engineering Science 220(7) (2006) 953-968.

[39] E. Simo-Serra, F. Moreno-Noguer, A. Perez-Gracia, Design of NonAnthropomorphic Robotic Hands for Anthropomorphic Tasks, in: Proc. of the 2011 ASME International Design Engineering Technical Conferences, Washington D.C., USA. 
[40] F. R. E. Crossley, A cotribution to grubler's theory in the number synthesis of plane mechanisms, Transactions of the ASME Journal of Engineering for Industry (1964) 1-8.

[41] R. M. Murray, Z. Li, S. S. Sastry, A Mathematical Introduction to Robotic Manipulation, CRC Press, Inc., Boca Raton, FL, 1994.

[42] J. M. Rico, J. Gallardo, B. Ravani, Lie algebra and the mobility of kinematic chains, Journal of Robotic Systems 20 (2003) 477-499.

[43] P. Fanghella, C. Galletti, Metric relations and displacement groups in mechanism and robot kinematics, Journal of Mechanical Design 117 (1995) 470-478.

[44] J. M. Rico, B. Ravani, On calculating the degrees of freedom or mobility of overconstrained linkages: Single-loop exceptional linkages, Journal of Mechanical Design 129 (2007) 301-311.

[45] E. Simo-Serra, A. Perez-Gracia, H. Moon, N. Robson, Design of Multifingered Robotic Hands for Finite and Infinitesimal Tasks using Kinematic Synthesis, in: J. Lenarcic, M. Husty (Eds.), Recent Advances in Robot Kinematics, Springer, 2012.

[46] A. Perez-Gracia, Synthesis of spatial rprp closed linkages for a given screw system, ASME Journal of Mechanisms and Robotics 3(2) (2011). 